\title{
Towards Pervasive Physical Rehabilitation Using Microsoft Kinect
}

\author{
Chien-Yen Chang ${ }^{1}$, Belinda Lange ${ }^{1}$, Mi Zhang ${ }^{2}$, Sebastian Koenig ${ }^{1}$, \\ Phil Requejo $^{3}$, Noom Somboon ${ }^{3}$, Alexander A. Sawchuk ${ }^{2}$, and Albert A. Rizzo ${ }^{1}$ \\ ${ }^{1}$ Institute for Creative Technologies, University of Southern California, Playa Vista, CA \\ ${ }^{2}$ Ming Hsieh Department of Electrical Engineering, University of Southern California, Los Angeles, CA \\ ${ }^{3}$ Rancho Los Amigos National Rehabilitation Hospital, Downey, CA \\ Email: kchang@ict.usc.edu, lange@ict.usc.edu, mizhang@usc.edu, skoenig@ict.usc.edu, \\ prequejo@larei.org,nsomboon@larei.org,sawchuk@sipi.usc.edu, rizzo@ict.usc.edu
}

\begin{abstract}
The use of Virtual Reality technology for developing tools for rehabilitation has attracted significant interest in the physical therapy arena. This paper presents a comparison of motion tracking performance between the low-cost Microsoft Kinect and the high fidelity OptiTrack optical system. Data is collected on six upper limb motor tasks that have been incorporated into a game-based rehabilitation application. The experiment results show that Kinect can achieve competitive motion tracking performance as OptiTrack and provide "pervasive" accessibility that enables patients to take rehabilitation treatment in clinic and home environment.
\end{abstract}

\section{INTRODUCTION}

In the United States, approximately 265,000 people have spinal cord injuries (SCI), resulting in partial or full paralysis [1]. People with such motor deficiencies experience dramatic limitations in performing everyday activities such as bathing, dressing and eating. Clinical studies have demonstrated that some motor deficiencies after SCI can be at least partially compensated for or recovered through physical rehabilitation. Conventional rehabilitation training programs typically involve extensive repetitive range-of-motion and coordination exercises and require professional therapists to supervise the patients' movements and assess the progress. However, this approach provides limited objective performance measurement and typically lacks engaging content to motivate individuals during the program.

To address these problems, new rehabilitation tools based on Virtual Reality (VR) and video games are being developed and have recently gained significant interest in the physical therapy arena. The core idea of VR-based rehabilitation is to use sensing devices to capture and quantitatively assess the movements of patients under treatment to track their progress more accurately. In addition, by integrating VR technology with video games, the goal is for patients to be more motivated and engaged in these physical activities.

Within the past decade, various motion capture systems have been developed and applied to rehabilitation research. The most precise technology uses reflective markers attached to the patients' bodies and limbs; they are tracked by optical sensors to determine their 3D position. However, its drawback is that the markers are often cumbersome and uncomfortable, inhibiting the patient's already limited movement and making these systems difficult to use in a clinical setting. In addition, they are also relatively expensive and typically housed at large medical facilities, requiring a patient visit and further increasing operational cost.

At the opposite end of complexity are relatively low-cost consumer game interface devices to infer mechanical motion. Popular examples include the Nintendo Wii Remote, the PlayStation Move, and the Microsoft Kinect. In this work, we propose a SCI rehabilitation system based on Microsoft Kinect. Kinect includes an RGB camera and a depth sensor, which together provide full-body 3D motion capture and joint tracking capabilities without markers or handheld controllers. Its user interface is nonintrusive and minimizes interaction with patient movement. Kinect is inexpensive, easy to set up, and can be used in both home and clinical environments. This "pervasive" accessibility could significantly facilitate rehabilitation, allowing more frequent repetition of exercises outside standard therapy sessions.

The objective of this paper is to investigate the capability of Microsoft Kinect as a robust tool for SCI rehabilitation. We experimentally compare the performance of a Kinectbased rehabilitation system with a high precision OptiTrack optical motion capture system. We then develop a prototype game for SCI rehabilitation and integrate it with our Kinectbased system to quantitatively assess patients' rehabilitation performance and track their progress in the clinic and home environment.

\section{RELATED WORK}

The use of VR technology for creating a new generation of tools for advancing rehabilitation has expanded rapidly over the past decade. In [2], the authors examined the feasibility of using Nintendo Wii (VRWii) for stroke rehabilitation and demonstrated that under the help of the VRWii system, participants had a significant improvement in motor function. In [3], the authors used the marker-based optical motion capture system Vicon for gait analysis on eighteen subjects with hemiparesis. Their experiment results showed that subjects using the Vicon system demonstrated a significantly larger increase in ankle power generation. Several studies identify the Kinect's potential for use in rehabilitation. For 
example, authors in [4] developed a Kinect-based system to assist therapists in rehabilitating students in public school settings. Their system could automatically determine whether the students' movements have reached the rehabilitation standard. As another example, authors in [5] integrated Kinect into a game-based rehabilitation task for balance training. Initial assessment of the prototype system with a sample of participants with neurological injury demonstrated that Kinect has potential as a rehabilitation tool.

Although existing work has shown the potential of Kinect as a useful VR rehabilitation tool, they did not rigorously examine the technical performance of Kinect. As will be shown in section IV, we experimentally validate that Kinect is not only convenient to use but also provides an acceptable level of quality of tracking performance.

\section{OUR SYSTEM}

In this section, we briefly describe the key technical features of the OptiTrack optical motion capture system and our Kinect-based rehabilitation system.

\section{A. OptiTrack Optical Motion Capture System}

The high performance optical motion capture system we used in this study is OptiTrack V100:R2 ${ }^{1}$. OptiTrack is a marker-based system which requires users to wear reflective markers such that their movements can be tracked by an array of cameras. OptiTrack is known for its high precision and processing capability. It can track markers down to submillimeter movements with repeatable accuracy and compute a skeleton model. In this work, we use the outputs of OptiTrack as ground truth and compare to the outputs of Kinect to validate whether Kinect can be used as a solid tool to develop applications for physical rehabilitation.

\section{B. Microsoft Kinect}

The Microsoft Kinect is a motion sensing input device developed as a peripheral device for use with the Xbox 360 gaming console ${ }^{2}$. Compared to the OptiTrack system, Kinect uses only one RGB camera combined with an infrared-based 3D depth sensor for full-body 3D motion capturing and joint tracking. Furthermore, Kinect enables users to control and interact with the console without the need to attach markers on the body. This "marker-free" user interface makes it a perfect VR tool for our rehabilitation application. A full comparison of technical specifications between Microsoft Kinect and OptiTrack is shown in Table I.

Kinect was originally designed only for use with the Xbox 360 console. To enable Kinect to be used with PC, we use the OpenNI/NITE ${ }^{3}$ middleware to read sensor data from Kinect. One of the benefits of using OpenNI/NITE is that it not only outputs raw Kinect camera and depth sensor data, but also supports user identification, scene segmentation, and skeleton/joint tracking. These advanced features are extremely

\footnotetext{
${ }^{1} \mathrm{http} / / /$ www.naturalpoint.com/optitrack/products/v100-r2/

${ }^{2}$ http://www.xbox.com/en-US/kinect

${ }^{3} \mathrm{http}: / /$ www.openni.org/
}

\begin{tabular}{|c|c|c|}
\hline & Microsoft Kinect & OptiTrack \\
\hline Resolution & $640 \times 480$ & $640 \times 480$ \\
\hline Frame Rate & 30 FPS & 100 FPS \\
\hline Sensing Range & 1.2 to $3.5 \mathrm{~m}$ & $20 \mathrm{~m}$ \\
\hline Field of View & $\begin{array}{c}\text { Horizontal: } 57^{\circ} \\
\text { Vertical: } 43^{\circ}\end{array}$ & $360^{\circ}$ \\
\hline Number of Cameras & 1 & Multiple \\
\hline Markers & No & Yes \\
\hline Cost & Low & High \\
\hline Environment & Clinic, Home & Medical Center \\
\hline
\end{tabular}

TABLE I

COMPARISON BETWEEN Microsoft KineCt AND OPTITRACK SYSTEM

useful for clinicians and therapists to assess the performance of their patients and to track their improvement. Therefore, we leverage these features to design our game that helps with SCI rehabilitation. Furthermore, we build a 3D avatar model as the human computer interface. The patients can control the avatar to interact in the game. The details of the game will be presented in the next section.

\section{Evaluation}

In this section, we describe the experiment and a rehabilitation game prototype we design for performance validation of our Kinect-based rehabilitation system.

\section{A. Experimental Setup}

We carried out the experiment at the Rancho Los Amigos National Rehabilitation Center Rehabilitation Engineering Laboratory. Two participants (one male with SCI and one healthy female) were recruited to take part in the study. Each participant followed the instructions from a professional therapist and was asked to perform six different types of motor tasks: (1) External Rotation; (2) Shoulder Adduction (Diagonal Pull Down); (3) Scapular Retraction; (4) Shoulder Abduction; (5) Shoulder Flexion; and (6) Shoulder Extension (see Table II for detailed explanations). Each task was repeated three times by each participant. These motor tasks are all targeting upper limb movement and are frequently employed in SCI therapy and diagnostics [6].

\begin{tabular}{|c|c|}
\hline Motor Task & \begin{tabular}{c} 
Description \\
\hline External Rotation
\end{tabular} \\
\hline $\begin{array}{c}\text { Shoulder Adduction } \\
\text { (Diagonal Pull Down) }\end{array}$ & $\begin{array}{c}\text { Shoulder flexion and adduction from arm above } \\
\text { head across midline of body towards opposite } \\
\text { knee with elbow extended }\end{array}$ \\
\hline Scapular Retraction & $\begin{array}{c}\text { Arms extended in front of body at 45 degrees } \\
\text { flexion, elbows extended - flexing elbows } \\
\text { to bring hands back towards body }\end{array}$ \\
\hline Shoulder Abduction & Shoulder abduction with elbow extended \\
\hline Shoulder Flexion & Shoulder flexion with elbow extended \\
\hline Shoulder Extension & $\begin{array}{c}\text { Arm outstretched in front of body with shoulder at } \\
90 \text { degrees flexion, elbow extended to arm outstretched } \\
\text { at side at 90 degrees abduction, elbows extended }\end{array}$ \\
\hline
\end{tabular}

TABLE II

MOTOR TASKS CONSIDERED IN OUR STUDY

When performing the experiment, each participant was wearing 20 markers on the upper body. The participant was seated on a chair facing the Kinect sensor at an approximate distance of 2.2 meters (Figure 1(b) shows marker placements). Meanwhile, 16 OptiTrack cameras mounted on the ceiling captured the movements simultaneously (see Figure 1(a)). 


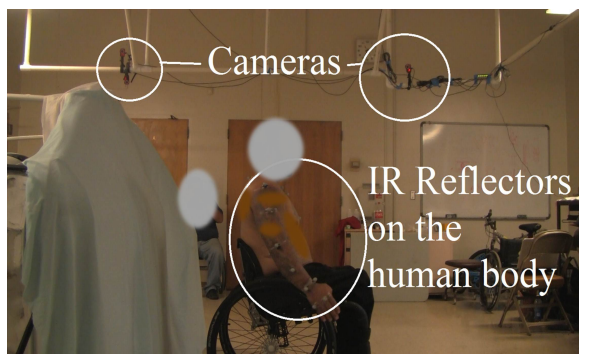

(a) The deployment of the OptiTrack cameras

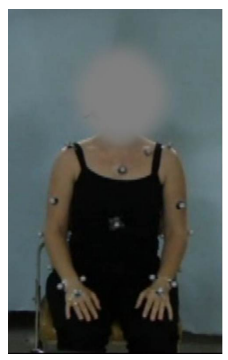

(b) The placement of the reflective markers on the upper body
Fig. 1. Experimental setup

\section{B. Comparison of Microsoft Kinect and OptiTrack}

1) Trajectory Comparison: We first compare the coordinate outputs of the Kinect and OptiTrack. Since these two devices use different coordinate systems, we transform the coordinates of OptiTrack into the coordinate system of Microsoft Kinect to make sure that all the three axes and the original points are matched. To make the comparison more meaningful to our rehabilitation application, we extract the joint information from the raw sensing data from both devices and compare the trajectories of the joints in 3D space. As our preliminary results, here we only illustrate the trajectories of the joints at right hand, right elbow, and right shoulder when performing motor task (1) External Rotation (Figure 2), (3) Scapular Retraction (Figure 3), and (4) Shoulder Abduction (Figure 4), respectively. As shown, for all three motor tasks, the tracking of hand and elbow are closely matched in each axis between Kinect (blue dotted line) and OptiTrack (red curve). However, the three movements at the shoulder are not tracked well by Kinect. One possible reason is that Kinect is placed in front of the participants and only uses one camera to track movements from one position. The movements occurring at the shoulder joint incur rotation, a movement can not be accurately captured by Kinect in the front view. In comparison, OptiTrack employs multiple cameras to track movements from multiple views, and thus captures most of the rotation movements at the shoulder joint. Our experiment results indicate that further exploration is needed to determine if the Kinect can accurately track rotation movement at the shoulder joint. However, our findings support that the Kinect can achieve competitive movement tracking performance as the high performance optical motion capture system like OptiTrack for hand and elbow joints tracking when Kinect is placed in front of the participants.

2) Timing Performance Comparison: Besides comparing the trajectory coordinates, we also evaluate the timing performance of both Kinect and OptiTrack. Specifically, we measure the relative latency between the outputs of these two systems when participants perform the movements. We use cross-correlation as the evaluation metric, which has been widely used as a standard method to find the time-lag between two time series. Our experiment shows that OptiTrack is 50 milliseconds faster than Kinect. This difference is negligible for our rehabilitation application.

\section{Game Design and Evaluation}

To further demonstrate Kinect as a solid VR rehabilitation tool, we implemented a game-based rehabilitation application using Unity3D game engine ${ }^{4}$. As an initial trial, our game only focuses on the motor task External Rotation. Before the game starts, the clinician can define the amount and the accuracy of the motor task by defining the starting point and end point of the motor task in terms of the location coordinates. As a necessary calibration step, the patient is asked to rotate his/her upper limb as much as possible such that the maximum rotation angle is recorded. Figure 5 shows a sequence of the screenshots of the game. During the game, the patient is encouraged to use his/her hand to move the virtual object from one side of the screen to the other. This exercise is often performed incorrectly, with the most common error being the movement of the elbow away from the body during the external rotation movement and the full movement not being completed as the number of repetitions increases. During the standard method of performing this exercise, the patients are not provided with any quantitative feedback on their performance and could potentially injure themselves. In comparison, our game keeps track of the location coordinates of the elbow and provides real time feedback on the quality of the movement through a visualization of the path of the hand movement. Specifically, when the patient exercises with the correct movement pattern, the path color is green. On the other hand, if the patient begins to move outside the required range, or his/her body position changes (such as the elbow moving away from the side of the body or the trunk leaning to one side to assist the movement), the path becomes red and arrows appear on the screen to guide the player back into the correct position. Finally, when the patient performs the correct movement and the hand reaches the pre-recorded maximum external rotation, the virtual object will be released from the patient's hand and the patient will be encouraged to return to the starting position to collect another object and perform the movement again.

To evaluate the performance of the game, we asked the two participants to perform the motor task External Rotation 10 times each, with 5 correct movements and 5 incorrect movements. Our game successfully identified all the incorrect movements by displaying the path in red color.

\section{CONCLUSiOn AND Future WORK}

This paper demonstrates the initial analysis and comparison of the motion tracking between the low-cost Microsoft Kinect and a high-cost multi-camera lab-based system OptiTrack. This comparison is an important aspect of the development of low-cost game-based VR rehabilitation tools. Based on our experiments, Microsoft Kinect is a promising VR neurological rehabilitation tool for use in the clinic and home environment. Future studies should address the comparison of more movement tasks involved in the rehabilitation interventions with a larger sample of participants with neurological injury.

\footnotetext{
${ }^{4}$ http://unity3d.com/
} 

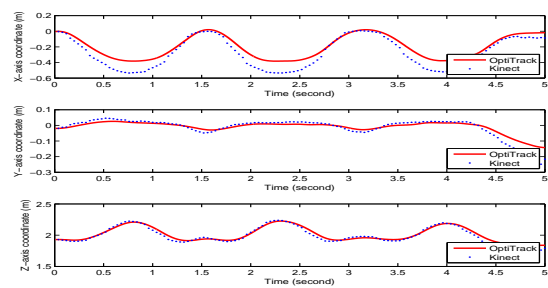

(a) The $\mathrm{x}, \mathrm{y}$, and $\mathrm{z}$-axis trajectories of right hand
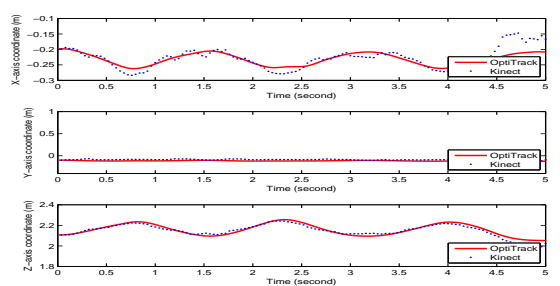

(b) The $\mathrm{x}, \mathrm{y}$, and $\mathrm{z}$-axis trajectories of right elbow
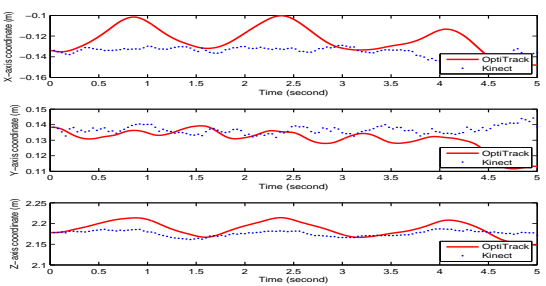

(c) The $\mathrm{x}, \mathrm{y}$, and $\mathrm{z}$-axis trajectories of right shoulder

Fig. 2. The trajectories of three joints at right hand, right elbow, and right shoulder when performing motor task External Rotation
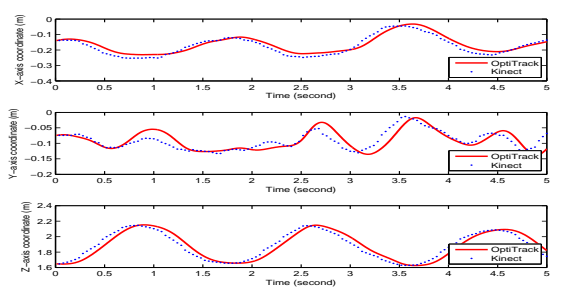

(a) The $\mathrm{x}, \mathrm{y}$, and $\mathrm{z}$-axis trajectories of right hand
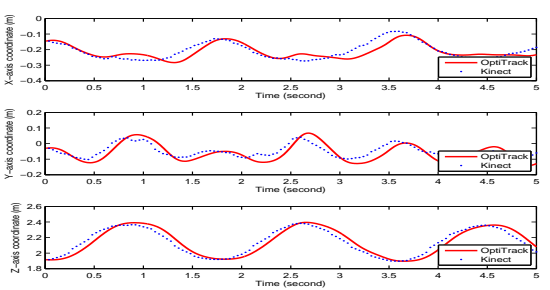

(b) The $\mathrm{x}, \mathrm{y}$, and $\mathrm{z}$-axis trajectories of right elbow
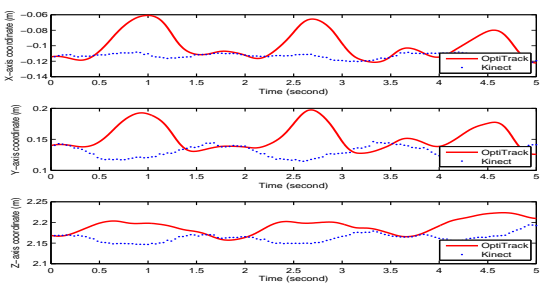

(c) The $x, y$, and z-axis trajectories of right shoulder

Fig. 3. The trajectories of three joints at right hand, right elbow, and right shoulder when performing motor task Scapular Retraction
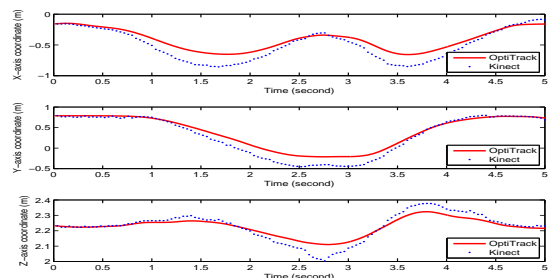

(a) The $\mathrm{x}, \mathrm{y}$, and $\mathrm{z}$-axis trajectories of right hand
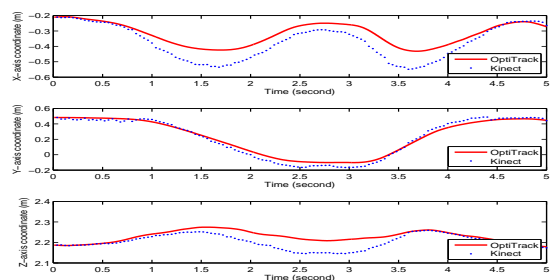

(b) The $\mathrm{x}, \mathrm{y}$, and $\mathrm{z}$-axis trajectories of right elbow
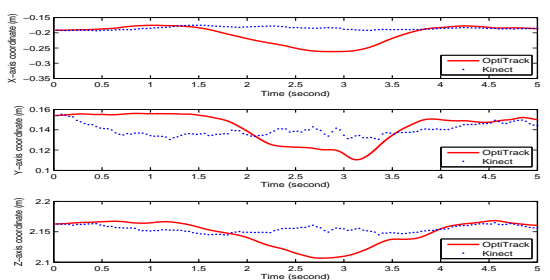

(c) The $\mathrm{x}, \mathrm{y}$, and z-axis trajectories of right shoulder

Fig. 4. The trajectories of three joints at right hand, right elbow, and right shoulder when performing motor task Shoulder Abduction

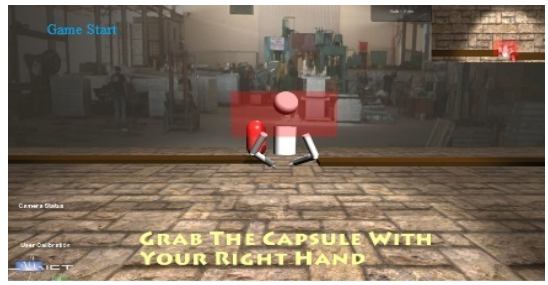

(a) At the beginning of the game, the player is ( instructed to grab the capsule using right hand

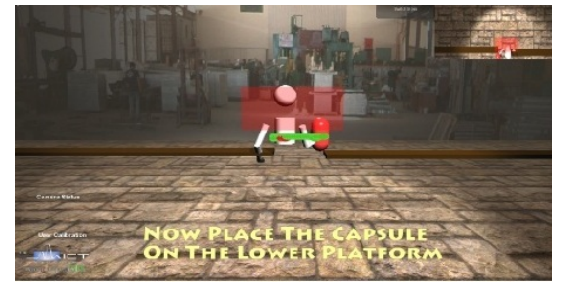

(b) The green color indicates the player's movement is correct

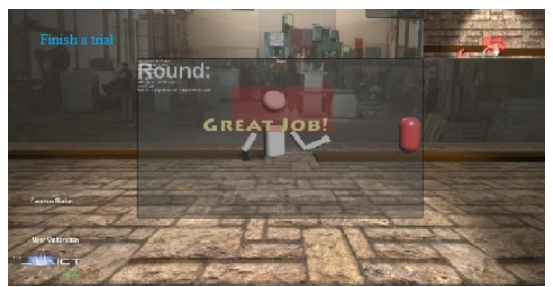

(c) The player successfully finishes the movement

Fig. 5. A sequence of screenshots of our rehabilitation game

\section{ACKNOWLEDGMENT}

This study was supported by a grant from the National Institute on Disability and Rehabilitation Research through the Rehabilitation Engineering Research Center (RERC) on Aging With a Disability (grant H133E080024).

\section{REFERENCES}

[1] National Spinal Cord Injury Statistical Center. Spinalcord injury facts and figures at a glance. Birmingham, Alabama, February 2011.

[2] Gustavo Saposnik et al. Effectiveness of virtual reality using Wii gaming technology in stroke rehabilitation: a pilot randomized clinical trial and proof of principle. Stroke, 41(7):1477-1484, 2010.
[3] Anat Mirelman, Benjamin L Patritti, Paolo Bonato, and Judith E Deutsch. Effects of virtual reality training on gait biomechanics of individuals poststroke. Gait \& Posture, 31(4):433-437, 2010.

[4] Yao-Jen Chang, Shu-Fang Chen, and Jun-Da Huang. A kinect-based system for physical rehabilitation: A pilot study for young adults with motor disabilities. Research in Developmental Disabilities, 32(6):25662570, 2011.

[5] Belinda Lange, Chien-yen Chang, Evan Suma, Bradley Newman, Albert Skip Rizzo, and Mark Bolas. Development and evaluation of low cost game-based balance rehabilitation tool using the microsoft kinect sensor. In International Conference of the Engineering in Medicine and Biology Society, pages 1831-1834, Boston, Massachusetts, August 2011.

[6] Sara J Mulroy et al. Strengthening and optimal movements for painful shoulders (STOMPS) in chronic spinal cord injury: a randomized controlled trial. Physical Therapy, 91:305-324, 2011. 